Editorial

\title{
Sensors in Connected Vehicle Technology: How Sensors Play a Critical Role
}

\author{
Chuan Ding, ${ }^{1}$ Jia $\mathrm{Hu}^{2}$ and Xiaolei $\mathrm{Ma}^{1}$ \\ ${ }^{1}$ School of Transportation Science and Engineering, Beihang University, Beijing 100191, China \\ ${ }^{2}$ Federal Highway Administration, Washington, DC, USA \\ Correspondence should be addressed to Chuan Ding; cding@buaa.edu.cn
}

Received 13 November 2017; Accepted 13 November 2017; Published 28 November 2017

Copyright (c) 2017 Chuan Ding et al. This is an open access article distributed under the Creative Commons Attribution License, which permits unrestricted use, distribution, and reproduction in any medium, provided the original work is properly cited.

A rapid development of connected vehicle (CV) technology has been observed around the globe in the past decade. The problems that the conventional traffic management systems bear are believed to be solved by introducing the technology. This CV technology puts diagnostic sensors onto vehicles/ infrastructures and has the data collected transmitted wirelessly between vehicles and nearby infrastructures. It would no longer rely on conventional data collection equipment, like a loop detector or video detections, and it collects much more information than the conventional ways. Measurements that are previously unknown are available, which include but are not limited to vehicle speeds, positions, arrival rates, rates of acceleration and deceleration, queue lengths, and stopped time. With this extra information, many applications are made possible. However, on the other hand, the accuracy and the stability of sensors cause challenges for the development of CV application, for instance, the accuracy of GPS, communication delay, and LIDAR accuracy. These factors require special design embedded in those CV applications to accommodate the limitation of sensors. This special issue aims to serve as a major platform to facilitate the discussion and exchange of research ideas and technology development, encourage multidimensional knowledge sharing, and enhance research activities in investigating sensors in connected vehicle technology. In total, seven papers are included in this special issue and are summarized as follows.

There are several articles focusing on traffic control and analysis using the sensor-based data to improve traffic system. The autonomous vehicle is able to facilitate road safety and traffic efficiency and has become a promising trend of future development. W. Wu et al. proposed traffic control models based on cellular automata for intersections in the autonomous vehicle environment. Multiagent technology is used to simulate the proposed model. The simulation results show that the control strategy of the proposed model significantly reduces average delays and a number of stops as well as increasing traffic capacity. A classical control problem for an isolated oversaturated intersection is revisited with a focus on the optimal control policy to minimize total delay. J. Tan et al. used the gradient descent algorithm to convert the optimal control plan of the continuous-time model to the plan of the discrete-time model in many cases. Analytic proof and numerical tests for the algorithm are also presented.

From the perspective of sensor-based traffic operation and management, several articles analyzed the traffic condition and driver behavior. With the development of connected vehicle $(\mathrm{CV})$ and vehicle to $\mathrm{X}(\mathrm{V} 2 \mathrm{X})$ communication, more traffic data are being collected from the road network. In order to predict future traffic condition from connected vehicles' data in real time, $\mathrm{P}$. Wang et al. proposed an online traffic condition evaluation model utilizing V2X communication. The contemporary vehicle data from the on-board diagnostic (OBD) is fused with the static road data in the roadside unit (RSU). Compared with traditional evaluation systems, the proposed model can handle more types of data but demands less data transfer. W. Zhao et al. did a sensorbased visual effect evaluation of chevron alignment signs' colors on drivers through the curves in snow and ice environment. The conclusions provide evident references for freeway warning products and the design of intelligent vehicles. 
There are several articles focusing on driving and travel behavior using the GPS data. J. Wang and Y. Cao developed a method to identify whether there is deliberate speed-up or slow-down movement of a bus based on the recovery of GPS data. The effectiveness of the developed method was demonstrated using the data collected in Harbin, China. The results show that it can help bus enterprises to design reasonable time of day intervals and significantly improve their level of service. S. An et al. proposed a public transit riders' travel pattern measuring method based on divided cells and public transit vehicle's GPS data. A case study is carried out to evaluate the methods, which use the GPS data collected from taxis and buses in Harbin, China. The study is expected to provide a better understanding of public transit riders' travel patterns.

The remaining one article investigated spatial crowdsourcing problem caused by the increasingly widespread sensors. With the development of sensors, particularly vehicle sensors and mobile sensors, spatial crowdsourcing is gaining even more attention. B. Du et al. proposed a novel spatial crowdsourcing problem called software development team formation (SDTF). They proved that SDTF was NP-hard and designed three greedy algorithms and an index-based algorithm to solve the SDTF problem. Extensive experiments are conducted on synthetic and real datasets, and the results verify the effectiveness and efficiency of the algorithms.

\section{Acknowledgments}

The guest editors hope the information provided in this special issue is useful. Finally, we would like to thank the authors for an excellent contribution of their research works and very warmly acknowledged the reviewers for their valuable review comments.

Chuan Ding $\mathrm{Jia} \mathrm{Hu}$

Xiaolei Ma 


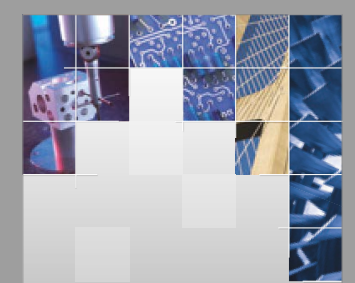

\section{Enfincering}
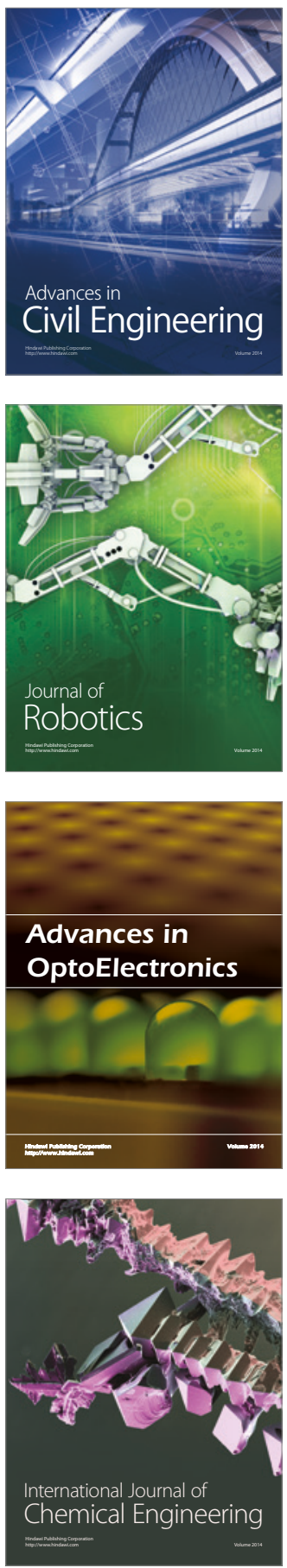

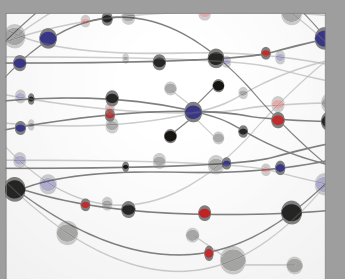

The Scientific World Journal

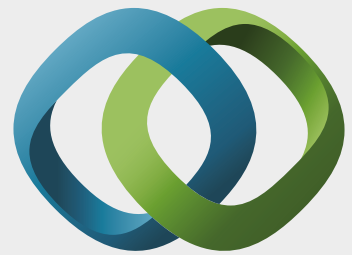

\section{Hindawi}

Submit your manuscripts at

https://www.hindawi.com
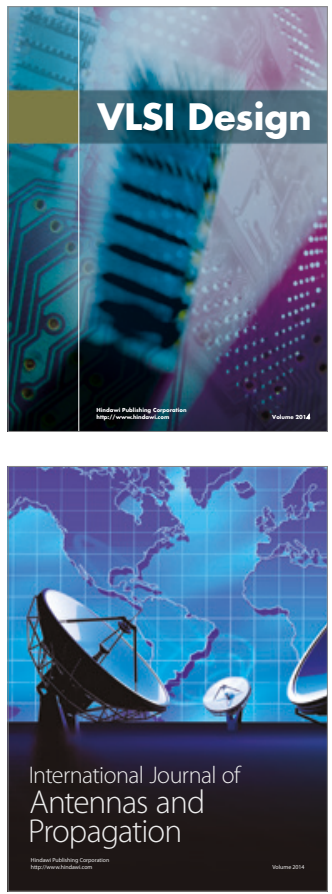

\section{Rotating}

Machinery
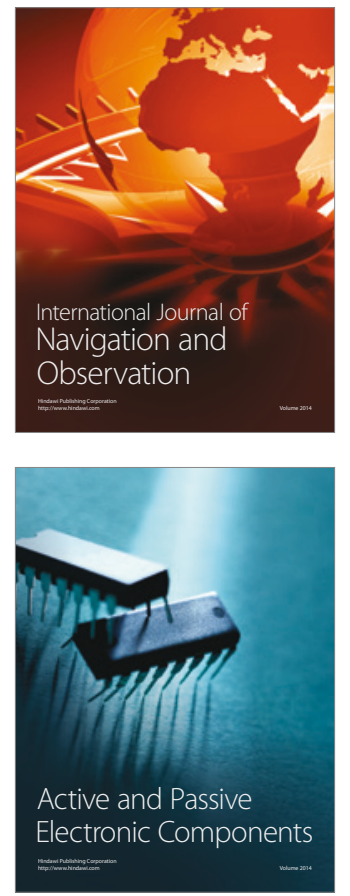
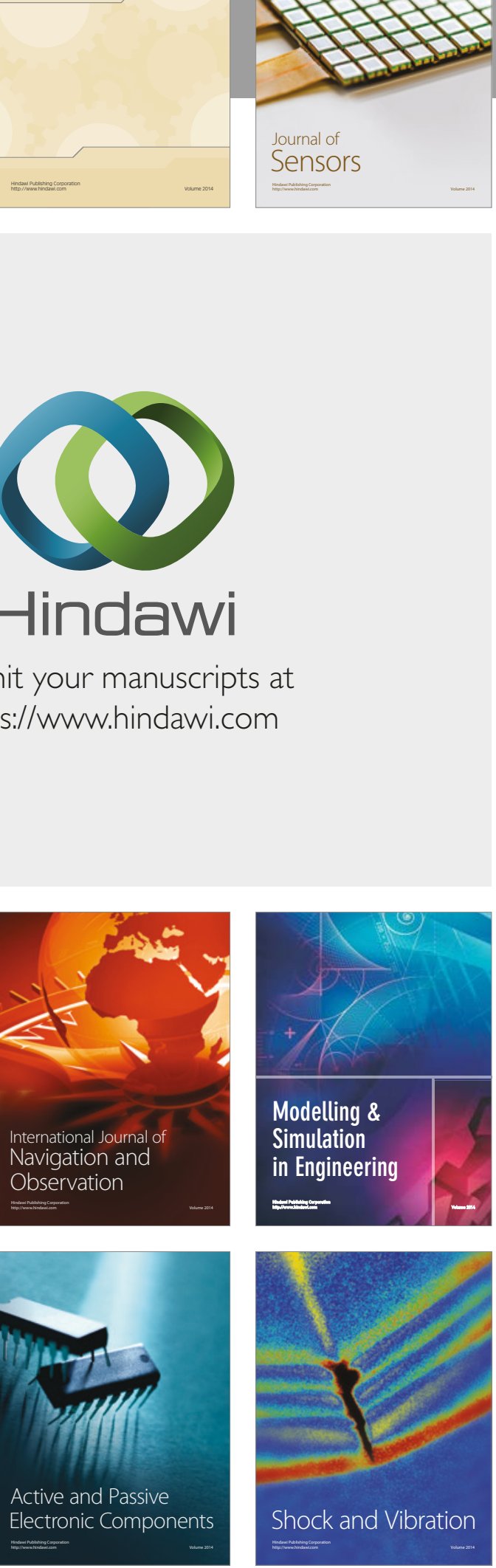
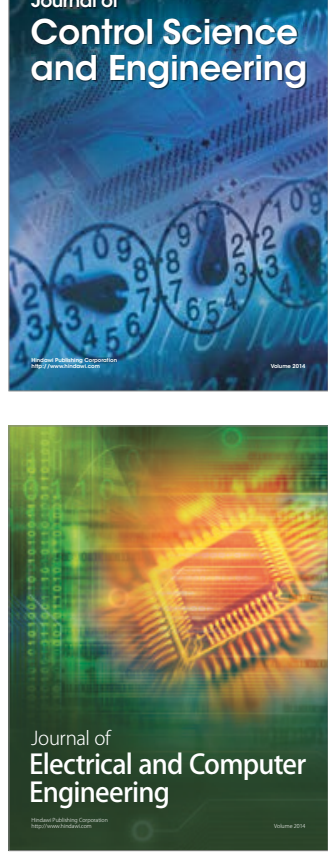

Distributed

Journal of

Control Science

and Engineering
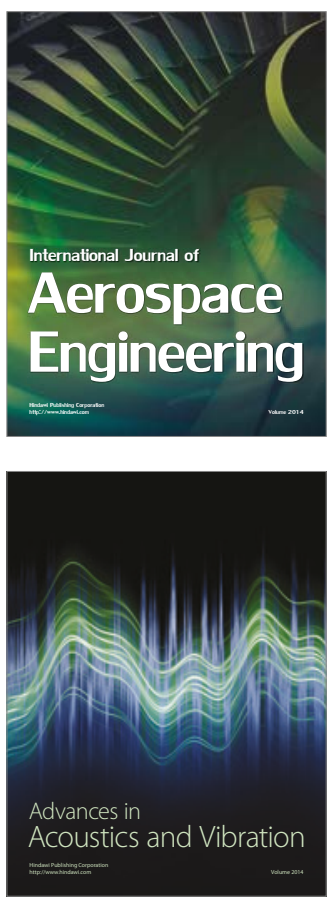

Sensor Networks 